\title{
Blood-based DNA methylation in Crohn's disease and severity of intestinal inflammation
}

\author{
Rahul Kalla ${ }^{1}$, Alex T. Adams ${ }^{2}$, Jack Satsangi ${ }^{2}$ \\ ${ }^{1}$ MRC Centre for Inflammation Research, Queens Medical Research Institute, University of Edinburgh, Edinburgh, UK; ${ }^{2}$ Translational \\ Gastroenterology Unit, Nuffield Department of Medicine, Experimental Medicine Division, University of Oxford, John Radcliffe Hospital, Oxford, \\ UK \\ Correspondence to: Professor Jack Satsangi. Lee Placito Professor of Gastroenterology, Translational Gastroenterology Unit, Nuffield Department of \\ Medicine, University of Oxford, Oxford, UK. Email: jack.satsangi@ndm.ox.ac.uk; j.satsangi@ed.ac.uk. \\ Provence: This is an invited article commissioned by the Guest Section Editor Dr. Li Min [Assistant Professor, Department of Gastroenterology, \\ Beijing Friendship Hospital (National Clinical Research Center of Digestive Disease), Capital Medical University, Beijing, China]. \\ Comment on: Somineni HK, Venkateswaran S, Kilaru V, et al. Blood-Derived DNA Methylation Signatures of Crohn's Disease and Severity of \\ Intestinal Inflammation. Gastroenterology 2019;156:2254-65.e3.
}

Received: 30 August 2019; Accepted: 08 October 2019; Published: 26 November 2019.

doi: $10.21037 / \operatorname{tgh} .2019 .10 .03$

View this article at: http://dx.doi.org/10.21037/tgh.2019.10.03

Considerable progress has been made over the last decade in the application of genome-wide association studies (GWAS) to identify potential susceptibility loci which may contain genes predisposing to inflammatory bowel disease (IBD). With over 200 identified, there are now ongoing efforts to identify the susceptibility genes associated with these loci, and the mechanisms involved in gene-environmental interactions.

It is now clear that germline variation only explains approximately $20 \%$ of the disease variance. Beyond genetics, studies are now exploring epigenetic mechanisms that associate with disease, DNA methylation being the most studied (1-7). The paper by Somineni et al. is a further contribution to the ever-advancing understanding of the methylome in IBD (6). DNA methylation profiles in whole blood were examined in 164 newly diagnosed paediatric CD at diagnosis and at 1-3 years of follow-up. These profiles were compared with age and sex matched controls (6). This study was part of the RISK registry; the consortium investigating molecular changes that define an aggressive disease behaviour over time in childhood-onset $\mathrm{CD}$, which has recruited an inception cohort across 28 centres in USA and Canada.

A number of points are worthy of discussion. Most importantly, there was strong independent replication of European/UK data signals such as RPS6KA2, SBNO2 and ITGB2, previously identified in UK adult and paediatric inception IBD cohorts $(2,3)$, across North America and
Canada. Replication at this scale across several populations is very encouraging, and indeed unique in methylation studies of complex diseases. This is a substantial progress in our understanding of epigenetic mechanisms at play in IBD at onset.

One of the key issues in DNA methylation studies is to decipher whether the changes seen are causal or as a consequence of disease. In order to unravel this, the authors carried out a number of analyses including correlation analyses with established markers of inflammation, longitudinal dynamics of DNA methylation. There was evidence of strong correlation with CRP ( $r=0.91$, $\left.\mathrm{P}<2.2 \times 10^{-16}\right)$ and most of these methylation changes reverted to levels seen in non-IBD controls at follow up. These changes did not associate with disease progression or medication exposure.

Mendelian randomization (MR) was then applied to the dataset and the authors identified 3 CpGs that were influenced by a known single nucleotide polymorphism rs1819333, within a locus on chromosome 17 that harbors key genes implicated in disease pathology. This region has also been implicated by GWAS in IBD, suggesting that DNA methylation may act as a mediator for this GWAS locus in CD.

From these analyses it appears appropriate to conclude that the majority of methylation changes may be associated with inflammation, and may be secondary to inflammatory 
processes. However, the real importance may be in those alterations that are resilient to inflammation. Methylation across $10 \mathrm{CpG}$ sites remained unchanged at follow up in this study; and it is noteworthy that germline variation at these loci are known to be associated with IBD through GWAS. A number are of obvious relevance to inflammatory pathways. RORC, a key transcription factor for the T-helper cell type 17 pathway, has been shown to be differentially expressed in peripheral blood and intestinal tissue in CD (8). Of all the genes identified, arguably the most worthy of further analysis is RPS6KA2, within the locus on chromosome 17 identified as causal by MR. In previous studies from UK and Europe, this gene has been shown to be significantly hypomethylated in IBD across several independent paediatric and adult cohorts $(2,3,9)$. RPS6KA2 encodes for a ribosomal kinase, a member of the serine/threonine kinase family and regulates important cell functions such as growth, motility and proliferation (10). More importantly, RPS6KA2 regulates the autophagy associated mTOR pathway and particularly relevant in CD pathogenesis (11).

In this post-GWAS era, studies are now beginning to unravel novel molecular mechanisms that underlie disease onset and progression $(9,12)$. The study of epigenetics provides a platform to explore the early molecular events that underlie disease pathogenesis, and gene-environmental interactions. This study has moved the field forward, firstly by showing strong independent replication in children in North America, of previous EWAS hits discovered in Northern Europe; and secondly by highlighting a small proportion of these alterations that may be resilient to changes in inflammatory activity. Future studies are now needed to explore the contribution of these epigenetic alterations in mediating gene-environment interactions, the potential role of methylation signals as a biomarker for disease, and in discovering new targets for future therapies.

\section{Acknowledgments}

The authors acknowledge funding support from CCUK, and from the European Commission.

\section{Footnote}

Conflicts of Interest: The authors have no conflicts of interest to declare.

Ethical Statement: The authors are accountable for all aspects of the work in ensuring that questions related to the accuracy or integrity of any part of the work are appropriately investigated and resolved.

\section{References}

1. Ventham NT, Kennedy NA, Nimmo ER, et al. Beyond gene discovery in inflammatory bowel disease: the emerging role of epigenetics. Gastroenterology 2013;145:293-308.

2. Ventham NT, Kennedy NA, Adams AT, et al. Integrative epigenome-wide analysis demonstrates that DNA methylation may mediate genetic risk in inflammatory bowel disease. Nat Commun 2016;7:13507.

3. Adams AT, Kennedy NA, Hansen R, et al. Two-stage genome-wide methylation profiling in childhood-onset Crohn's Disease implicates epigenetic alterations at the VMP1/MIR21 and HLA loci. Inflamm Bowel Dis 2014;20:1784-93.

4. Howell KJ, Kraiczy J, Nayak KM, et al. DNA Methylation and Transcription Patterns in Intestinal Epithelial Cells From Pediatric Patients With Inflammatory Bowel Diseases Differentiate Disease Subtypes and Associate With Outcome. Gastroenterology 2018;154:585-98.

5. McDermott E, Ryan EJ, Tosetto M, et al. DNA Methylation Profiling in Inflammatory Bowel Disease Provides New Insights into Disease Pathogenesis. J Crohns Colitis 2016;10:77-86.

6. Somineni HK, Venkateswaran S, Kilaru V, et al. BloodDerived DNA Methylation Signatures of Crohn's Disease and Severity of Intestinal Inflammation. Gastroenterology 2019;156:2254-65.e3.

7. Harris RA, Nagy-Szakal D, Mir SA, et al. DNA methylation-associated colonic mucosal immune and defense responses in treatment-naïve pediatric ulcerative colitis. Epigenetics 2014;9:1131-7.

8. Maddur MS, Miossec P, Kaveri SV, et al. Th17 cells: biology, pathogenesis of autoimmune and inflammatory diseases, and therapeutic strategies. Am J Pathol 2012;181:8-18.

9. Kalla R, Adams A, Nimmo E, et al. Epigenetic alterations in inflammatory bowel disease: the complex interplay between genome-wide methylation alterations, germline variation, and gene expression. Lancet 2017;389:S52.

10. Maller JL, Foulkes JG, Erikson E, et al. Phosphorylation of ribosomal protein $\mathrm{S} 6$ on serine after microinjection of the Abelson murine leukemia virus tyrosine-specific protein kinase into Xenopus oocytes. Proc Natl Acad Sci 
U S A 1985;82:272-6.

11. Pancholi S, Lykkesfeldt AE, Hilmi C, et al. ERBB2 influences the subcellular localization of the estrogen receptor in tamoxifen-resistant MCF-7 cells leading to the activation of AKT and RPS6KA2. Endocr Relat Cancer

doi: $10.21037 / \operatorname{tgh} .2019 .10 .03$

Cite this article as: Kalla R, Adams AT, Satsangi J. Bloodbased DNA methylation in Crohn's disease and severity of intestinal inflammation. Transl Gastroenterol Hepatol 2019;4:76.
2008; 15:985-1002.

12. Olszak T, An D, Zeissig S, et al. Microbial exposure during early life has persistent effects on natural killer $\mathrm{T}$ cell function. Science 2012;336:489-93. 